\title{
Medicamentos homeopáticos e o paradigma da evidência científica
}

Homeopathic medicines and the scientific paradigm

Medicamentos homeopáticos y el paradigma de la evidencia científica

Carla Holandino'; Thaís de Barros Fernandes ${ }^{2}$; Camila Monteiro Siqueira ${ }^{3}$; Juliana Patrão de Paiva $^{4}$; Fortune Homsani ${ }^{5}$; Gleyce Moreno Barbosa ${ }^{6}$; Sheila Garcia ${ }^{7}$, Adriana Passos Oliveira ${ }^{8}$

\section{Resumo}

Medicamentos homeopáticos são toda forma farmacêutica de dispensação ministrada segundo o princípio da semelhança e/ou identidade, com finalidade curativa e/ou preventiva. O princípio hipocrático da semelhança foi experimentado por Hahnemann, que assim o definiu em 1796: "Toda substância que administrada em doses ponderais, até mesmo tóxica para o homem de boa saúde, desencadeia distúrbios precisos, torna-se, depois de diluída e agitada, capaz de induzir o desaparecimento desses mesmos distúrbios em indivíduos doentes". Este é considerado até os dias de hoje o grande paradigma homeopático e, no presente estudo, o discutimos com base na literatura acadêmico-científica. Os trabalhos realizados com diferentes modelos evidenciam que as altas diluições homeopáticas são soluções que possuem atividade farmacológica e que, portanto, não podem ser consideradas placebos. $\mathrm{O}$ cenário atual é bastante promissor, uma vez que a qualidade metodológica, assim como a compreensão acerca dos alvos celulares e moleculares disparados pelo estímulo homeopático, têm conferido status científico a esta terapêutica baseada em evidências. $\mathrm{O}$ avanço na compreensão dos mecanismos de ação depende de alguns desafios que precisam ser superados, tais como: aumento do número de pesquisadores interessados na compreensão dos sistemas dinamizados; ampliação dos modelos experimentais capazes de registrar alterações físicas, químicas e biológicas disparadas pelos medicamentos homeopáticos; e reprodutibilidade dos resultados experimentais. Estes e outros aspectos devem ser estimulados no ambiente acadêmico e profissional, a fim de romper as barreiras e os paradigmas que se contrapõem ao avanço desta Prática Integrativa e Complementar de Saúde.

Descritores: Homeopatia; Lei dos Semelhantes; Medicamento Homeopático; Dinamização.

\section{Abstract}

Homeopathic medicines are every pharmaceutical form of dispensing delivered according to the

\footnotetext{
${ }^{1}$ Doutor em Biofísica pela Universidade Federal do Rio de Janeiro (UFRJ). Professor Associado do Curso de Graduação em Farmácia. Av. Carlos Chagas Filho, 373, Cidade Universitária, Rio de Janeiro, RJ, Brasil. CEP: 21941-902. E-mail: cholandino@gmail.com

${ }^{2}$ Farmacêutica pela Universidade Federal do Rio de Janeiro (UFRJ).

${ }^{3}$ Doutor em Ciências Farmacêuticas pela Universidade Federal do Rio de Janeiro (UFRJ). Professor do Curso Técnico em Farmácia no Instituto Federal de Educação, Ciência e Tecnologia (IFECT).

${ }^{4}$ Mestre em Ciências Farmacêuticas pela Universidade Federal do Rio de Janeiro (UFRJ). Farmacêutica do Laboratório de Manipulação Homeopática da Farmácia Universitária da UFRJ.

${ }^{5}$ Mestre em Ciências Farmacêuticas pela Universidade Federal do Rio de Janeiro (UFRJ). Farmacêutica Assistente Técnica do Laboratório de Manipulação Homeopática da Farmácia Universitária da UFRJ.

${ }^{6}$ Doutor em Ciências Farmacêuticas pela Universidade Federal do Rio de Janeiro (UFRJ). Farmacêutica do Laboratório de Pesquisa \& Desenvolvimento de Práticas Integrativas e Complementares da UFRJ.

${ }^{7}$ Faculdade de Farmácia da Universidade Federal do Rio de Janeiro (UFRJ)

${ }^{8}$ Doutor em Química de Produtos Naturais pela Universidade Federal do Rio de Janeiro. Professor Adjunto do Curso de Graduação em Farmácia da Universidade Federal do Rio de Janeiro (UFRJ).
} 
ISSN 2179-6750

principle of similarity and/or identity, for curative and/or preventive purposes. The Hippocratic principle of similarity was tested by Hahnemann, who, in 1796, defined it as: "Every substance which is administered in heavy doses, even toxic to man in good health, triggers precise disturbances, becomes, after diluted and agitated, capable of inducing the disappearance of these same disorders in sick individuals ". To this day, this has beenthe great homeopathic paradigm and, in the present study, we discuss it based on the academic-scientific literature. Using different models, Literature shows that high homeopathic dilutions are solutions with pharmacological activity and, because of this, can not be considered as placebos. The current scenario is quite promising considering the high quality of methodological tools available, as well as the understanding of cellular and molecular targets triggered by homeopathic stimulation. These aspects have conferred scientific status to this evidence-based therapy. The advance in the understanding of homeopathic action mechanisms depends on some challenges that need to be overcome, such as: increasing the number of researchers interested in dynamized systems; expansion of experimental models capable of recording physical, chemical and biological changes triggered by homeopathic medicines; and reproducibility of the experimental results. These and other aspects should be stimulated in the academic and professional environment, in order to break down the barriers and paradigms that oppose the advancement of this Integrative and Complementary Health Practice.

Key-words: Homeopathy; Law of Similars; Homeopathic Remedy; Dynamization.

\section{Resumen}

Los medicamentos homeopáticos son toda forma farmacéutica de dispensación ministrada según el principio de la semejanza y/o identidad y que tienen finalidad curativa y/o preventiva.

El principio hipocrático de semejanza fue experimentado por Hahnemann, quien así lo definió en 1976: "Toda sustancia que, al ser administrada en dosis ponderadas, hasta mismo tóxicas para personas de buena salud, desencadena disturbios específicos, volviéndose, después de ser diluida y agitada, capaz de inducir el desaparecimiento de esos mismos disturbios en individuos enfermos". Este es considerado hasta los días actuales el gran paradigma de la homeopatía, y en el presente trabajo, es discutido con base en la literatura científica. Los trabajos realizados con diferentes modelos evidencian que las altas diluciones homeopáticas son soluciones que poseen actividad farmacológica y que por lo tanto no pueden ser considerador placebos. El escenario actual es bastante prometedor porque la calidad metodológica, así como la comprensión acerca de los lugares específicos celulares y moleculares identificados por el estímulo homeopático, han conferido status científico a esta terapia basada en evidencias. El avance en la comprensión de los mecanismos de acción depende de algunos desafíos que necesariamente deben ser superados, tales como: la ampliación de los modelos experimentales capaces de registrar las alteraciones físicas, químicas e biológicas provocadas por los medicamentos homeopáticos, y la reproductibilidad de los resultados experimentales. Estos y otros aspectos deben ser estimulados en el ambiente académico y profesional, con el fin de romper barreras y paradigmas que se contraponen al avance de esta Práctica Integradora y Complementar de la Salud.

Palabras-claves: Homeopatía; Ley de la Similitud; Medicamento Homeopático; Dinamización.

\section{Introdução}

Os medicamentos homeopáticos são toda forma farmacêutica de dispensação ministrada segundo o princípio da semelhança e/ou identidade, com finalidade curativa e/ou preventiva, segundo a Farmacopeia Homeopática Brasileira. Nesta definição, é encontrado o primeiro 
ISSN 2179-6750

paradigma da homeopatia: a lei da semelhança, descrita originalmente por Hipócrates, o pai da medicina, cerca de 400 anos antes da era Cristã. Nesta conjuntura extraordinária de grandes filósofos, Hipócrates descreveu os três grandes princípios curativos, também conhecidos como leis de cura das doenças, a saber: "Vis Natura Medicatrix" (via natural de cura das doenças); "Contraria Contraribus Curantur" (uso de medicamentos que induzem sintomas contrários à doença); "Similia Similibus Curantur" (a doença seria produzida pelos semelhantes e pelos semelhantes seria curada) 1

Muitos séculos após a era Hipocrática nasce, na cidade de Meissen (Alemanha), Cristiano Frederico Samuel Hahnemann (1755-1843). Personalidade marcada por uma aguçada inteligência e elevado espírito científico motivaram Hahnemann, desde muito cedo, ao estudo da medicina e da química. O nascimento oficial da terapêutica homeopática aconteceu no ano de 1796 quando Hahnemann publica no Journal of Practical Pharmacology and Surgery o artigo intitulado: "Essay on a new principle for discovering the healing virtues of medicinal substances, as well as some comments on those used to date" ${ }^{2}$. Nesta publicação, Hahnemann resgata o princípio hipocrático da semelhança e o transforma numa nova terapêutica baseada no uso de substâncias dinamizadas. Esta forma de preparo do medicamento homeopático é dita hahnemanniana, porque segue os preceitos farmacotécnicos originalmente desenvolvidos pelo "pai da homeopatia" com atualizações técnicas descritas nas farmacopeias e nos compêndios oficiais de homeopatia. Hahnemann experimentou na prática o princípio hipocrático da semelhança assim definido: “Toda substância que, administrada em dose forte, ponderável, até mesmo tóxica para o homem de boa saúde, desencadeia distúrbios precisos, torna-se, depois de diluída, portanto, em dose fraca, capaz de induzir o desaparecimento desses mesmos distúrbios em indivíduos doentes". Este é considerado o grande paradigma homeopático ${ }^{1}$.

\section{Métodos}

O presente estudo é descritivo com abordagem qualitativa da literatura científica que relata as evidências científicas atribuídas aos medicamentos homeopáticos. O levantamento bibliográfico foi realizado utilizando bases de dados online, como Scientific Eletronic Library Online (SciELO) e a Medical Publication (PubMed).

\section{Resultados e discussão}

De Hahnemann até os dias de hoje, os cientistas em todo o mundo buscam modelos experimentais que permitam comprovar o "Princípio da similitude". Para tal, vários experimentos 
ISSN 2179-6750

in vitro, in vivo e ensaios clínicos têm sido desenvolvidos. Apesar de não ser ainda exatamente conhecido como agem os medicamentos homeopáticos, há algumas teorias importantes, dentre as quais destacamos a que se baseia na transmissão da informação medicamentosa traduzida em diferentes níveis, como: celular, físico e químico. Dentre os vários trabalhos, encontram-se alguns clássicos como os publicados por Belon e colaboradores ${ }^{3}$, que evidenciam que a relação "chavefechadura" não pode ser comprovada nos experimentos homeopáticos, em contraposição aos modelos alopáticos tradicionais. Entretanto, utilizando células do sistema imune, como macrófagos, se verifica que estas soluções diluídas e dinamizadas são capazes de estimular a liberação de citocinas $^{4}$, apresentando uma atividade imunomoduladora facilmente comprovada também em sistemas complexos ${ }^{5-7}$. Outros efeitos sutis têm sido registrados, como a ativação de reações apoptóticas por medicamentos homeopáticos, as quais justificam a atividade antitumoral destas substâncias ${ }^{8}$.

Holandino e colaboradores vêm estudando as propriedades físico-químicas de soluções dinamizadas ${ }^{9,10}$, preparadas a partir de medicamentos de origem vegetal ${ }^{11}$, biológica ${ }^{12,13}$, sintética 10 e mineral ${ }^{14}$, em potências decimais ${ }^{4,13}$, centesimais e cinquenta milesimais ${ }^{11}$. Elia e colaboradores sugeriram que o envelhecimento das soluções dinamizadas implica em alterações físico-químicas, capazes de modificar a condutividade elétrica e outras propriedades, devido à geração de estruturas dissipativas ${ }^{15,16}$.

Outras teorias têm sido propostas na literatura sendo uma das primeiras descrita pelo pesquisador francês Jacques Benveniste ${ }^{17}$. A polêmica teoria da "memória da água", originalmente publicada por Benveniste e colaboradores e, posteriormente, por outros pesquisadores, postula que a água é capaz de guardar a "memória" da substância originalmente solubilizada neste meio aquoso. A transmissão desta memória parece estar envolvida com o processo de sucussão, uma vez que, na ausência das agitações, o efeito biológico é significativamente influenciado ${ }^{3,16-18}$. Adicionalmente, os trabalhos de Montaigner indicam que a água é capaz de adquirir propriedades eletromagnéticas decorrentes da dinamização, as quais podem ser amplificadas através de aparato desenvolvido e patenteado por Benveniste ${ }^{19}$.

Desta forma, verificamos que os trabalhos científicos realizados com diferentes modelos evidenciam que as altas diluições homeopáticas são soluções que possuem atividade farmacológica e que, portanto, não podem ser consideradas placebos ${ }^{4,12,20,21,22}$. Entretanto, o mecanismo pelo qual estas soluções, que não apresentam moléculas detectáveis do insumo ativo, são capazes de ativar o sensor biológico está longe de ser esclarecido. Neste cenário de consolidação científica da homeopatia, diferentes abordagens teóricas e experimentais são descritas, dentre as quais destacam- 
ISSN 2179-6750

se: a física quântica ${ }^{23}$, as medidas de condutividade elétrica e espectrométricas ${ }^{16,24}$, a termoluminescência ${ }^{18}$ dentre outros modelos. Atualmente, dois modelos principais destacam-se na literatura ${ }^{20}$ : os clatratos ou clusters de moléculas de água e os domínios coerentes postulados pela eletrodinâmica quântica (QED).

O modelo dos clatratos, ou agregados de moléculas de água ou clusters deram origem às primeiras teorias da "memória da água" ${ }^{25-30}$. Segundo este modelo, sob certas condições de agitação ou sonicação do líquido, diferentes figuras geométricas complexas, com conformações poligonais seriam originadas. Estas figuras, que podem estar internamente vazias, especialmente após a ultradiluição, se organizam a partir de diferentes ligações de hidrogênio e outras ligações químicas, como dipolos entre hidrogênio e íons hidroxila. De acordo com este modelo, um determinado número de moléculas da substância original seria solvatada pelo solvente, dando origem a nichos ou gaiolas ${ }^{28}$. Estas estruturas manteriam a sua estabilidade, mesmo quando a substância inicial fosse expelido do próprio nicho, em decorrência das diluições sucessivas. Assim, após as diluições e sucussões necessárias à produção das soluções homeopáticas, clatratos vazios seriam formados; mas estes, entretanto, seriam capazes de manter os núcleos equivalentes do padrão originalmente induzido pela substância ativa, utilizada no preparo das primeiras dinamizações homeopáticas (Figura 1).

Figura 1. Agregados, clusters ou gaiolas hídricas na forma de (A) Cluster icosaedral de moléculas de água e (B) Cluster icosaedral colapsado com dodecaedro central.
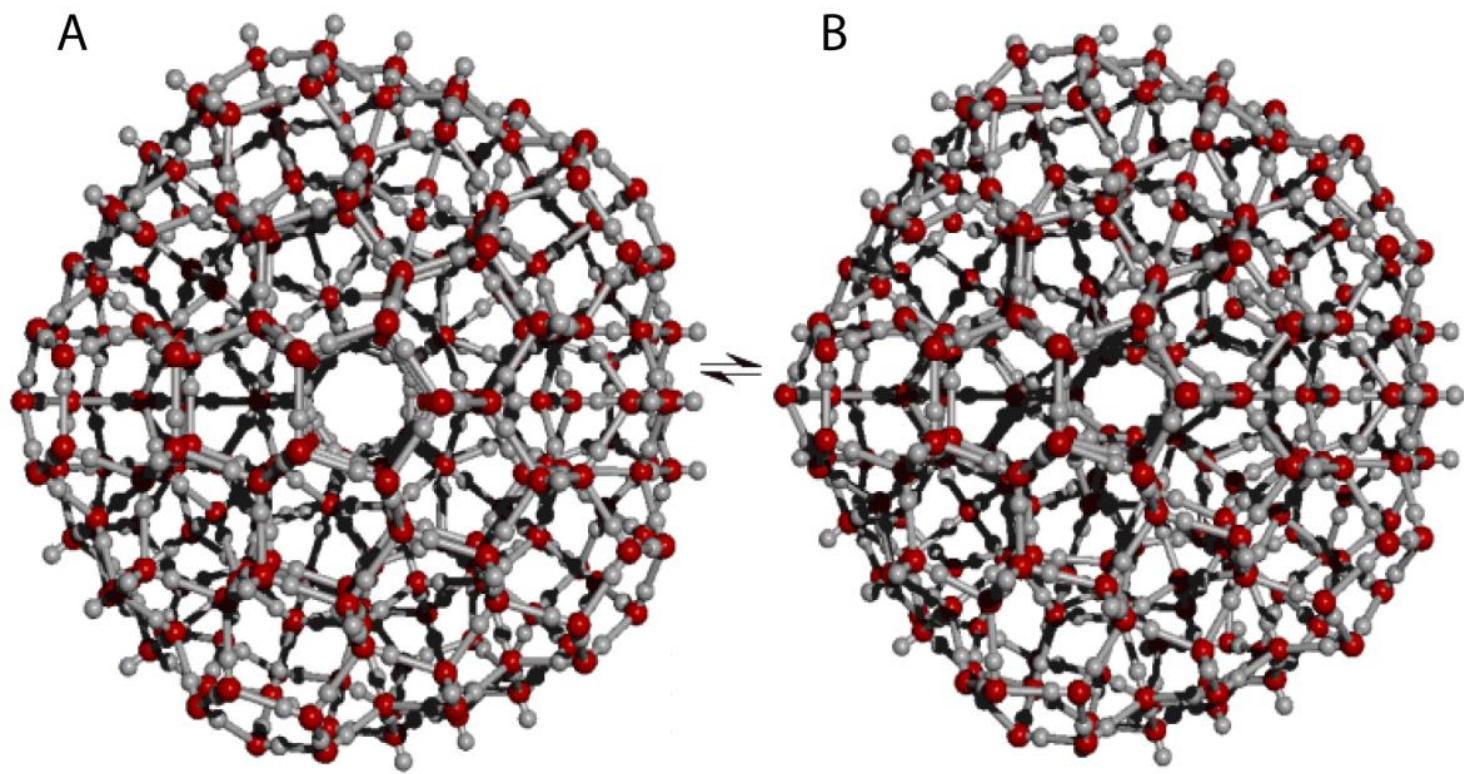

Fonte: Reproduzida com permissão de Chaplin ${ }^{28,30}$.

A teoria dos domínios coerentes (QED) postula a permanência de informações biologicamente ativas em estruturas compostas de muitas moléculas de água ou associadas a 
ISSN 2179-6750

soluções hidroetanólicas, tradicionalmente utilizadas nas farmácias homeopáticas, além da existência de domínios coerentes de água, cujos dipolos elétricos poderiam oscilar em fase ${ }^{28,20}$.

A formação de nanopartículas a partir da interação do soluto com o solvente também tem sido considerada na tentativa de compreender o comportamento das soluções ultradiluídas ${ }^{20,28-32}$. Todavia, esta hipótese precisa ser melhor estudada, uma vez que a existência de nanopartículas em sistemas líquidos e sólidos, ainda não é consenso na literatura ${ }^{20}$ Neste cenário, surge a atual necessidade do preparo dos medicamentos homeopáticos de acordo com rigoroso padrão, visando à garantia da qualidade e a reprodutibilidade dos resultados experimentais. Estes e outros aspectos têm sido investigados em um Projeto Multicêntrico onde Brasil e Itália vem trabalhando os aspectos físicos, químicos e biológicos do medicamento homeopático Zincum mettalicum ${ }^{14}$.

Outra questão a ser considerada diz respeito a físico química da água. Verificamos na prática que todo processo de purificação, seja por destilação, ultrafiltração ou autoclavação não consegue excluir completamente os "contaminantes" químicos da água ${ }^{14}$. Desta forma, verificamos que as características físico-químicas da água purificada variam consideravelmente e que a presença destes contaminantes tornam a água capaz de originar estruturas mais ou menos complexas, capazes de interagir química ou fisicamente com o meio dissolutor. Se não conseguimos explicar adequadamente as propriedades da água purificada mais complexo ainda se torna o estudo da água dinamizada ou sucussionada.

Um fenômeno que vem sendo descrito na água no estado líquido é a autotixotropia, no qual observa-se a formação de um gel fraco, produzido a partir da interação das moléculas de água entre si, mesmo em concentrações muito baixas de sais ${ }^{20,33}$. Curiosamente, tem-se argumentado que espécies ativas de oxigênio presentes na água devido a reações em cadeia desencadeadas por perturbações iniciais, mesmo na presença de diminutas quantidades de impurezas, devem ter um papel importante na manutenção dos estados de "não-equilíbrio" em sistemas aquosos ${ }^{30}$. Evidências importantes têm sido fornecidas por experimentos que envolvem a Ressonância Magnética Nuclear (RMN) onde gases dissolvidos na água parecem afetar a rede de ligações de hidrogênio e modificar a estrutura das nanobolhas ou das nanocavidades dos clatratos ${ }^{34}$. Além disso, a dinâmica de pequenos clusters de água é também marcadamente afetada pelo processo de ionização ${ }^{20,35}$. Neste contexto, tem sido sugerida a participação da sílica na formação de aglomerados de água; esta seria liberada a partir dos recipientes de vidro, utilizados normalmente na preparação dos medicamentos homeopáticos ${ }^{36}$. As nanoestruturas de sílica formadas durante a sucussão em vidro também poderiam adquirir e transmitir informações epitaxais obtidas a partir das tinturas mães ou de outros insumos ativos ${ }^{37}$. Se isto for verdade, o processo de dissolução da sílica 
ISSN 2179-6750

seria um fator chave para a formação de nanopartículas e, possivelmente, para o efeito medicamentoso das soluções homeopáticas, justificando a necessidade de se preparar as dinamizações sempre em recipientes de vidro e não em plástico. Entretanto, esta teoria precisa ser melhor comprovada. Alguns estudos ${ }^{38,39}$ reportam resultados positivos desencadeados pelos medicamentos homeopáticos, mesmo quando estes são preparados em frascos de plástico descartáveis, sugerindo que o uso de vidro pode não ser uma exigência absoluta para o aparecimento do efeito medicamentoso.

Neste cenário de consolidação científica da homeopatia, podemos dizer que: i) O fenômeno das ultradiluições homeopáticas foge do escopo da ciência tradicional; ii) O mecanismo de ação do medicamento homeopático não segue o modelo clássico "chave-fechadura"; iii) A água, solvente muito utilizado para o preparo das soluções homeopáticas, apresenta características físico-químicas anômalas que ainda não são completamente compreendidas à luz da ciência tradicional.

\section{Conclusão}

A comprovação do princípio hipocrático da semelhança, considerado o grande paradigma homeopático, poderá trazer uma nova compreensão acerca dos sistemas dinamizados. Entretanto, nos dias de hoje, os inúmeros resultados experimentais nos permitem destacar que: as ultradiluições homeopáticas geram soluções farmacologicamente ativas e, portanto, não podem ser consideradas placebos. O mecanismo pelo qual estas preparações são capazes de ativar o sensor biológico está, contudo, longe de ser completamente elucidado. O aumento da qualidade metodológica, assim como o avanço na compreensão dos fenômenos decorrentes do processo de dinamização, precisa ser fomentado para que esta ciência, relativamente nova, seja seriamente investigada. Este pode ser o caminho para que o paradigma dos sistemas dinamizados seja decifrado a luz da ciência tradicional e, consequentemente, nos permita avançar na compreensão dos mecanismos de ação dos medicamentos homeopáticos.

\section{Referências}

1. Corrêa AD, Siqueira-Batista R, Quintas LEM. Similia Similibus Curentur: notação histórica da medicina homeopática. Rev Assoc Med Bras (1992). 1997;43(4):347-51. http://dx.doi.org/10.1590/S0104-42301997000400013.

2. Hahnemann S. The Lesser Writings: Essay on a new principle for acertaining the curative powersof drugs, with a few glances at those hitherto employed. J praktischen Arzneykunde Hufeland. 1796;2(part 3):249-303. 
ISSN 2179-6750

3. Belon P, Cumps J, Ennis M, Mannaioni PF, Roberfroid M, Sainte-Laudy J. Histamine dilutions modulate basophil activation. Inflamm Res. 2004 May53(5):181-8. https://doi.org/10.1007/s00011-003-1242-0.

4. Siqueira CM, Costa B, Amorim AM, Gonçalves M, Veiga VF, Castelo-Branco M, et al. H3N2 homeopathic influenza virus solution modifies cellular and biochemical aspects of MDCK and J774G8 cell lines. Homeopathy. 2013 Jan;102(1):31-40. https://doi.org/10.1016/j.homp.2012.10.003.

5. Siqueira CM, Motta PD, Cardoso TN, Coelho CP, Popi AF, Couceiro JN, et al. Homeopathic treatments modify inflammation but not behavioral response to influenza antigen challenge in $\mathrm{BALB} / \mathrm{c}$ mice. Homeopathy. 2016 Aug;105(3):257-64. https://doi.org/10.1016/j.homp.2016.04.002.

6. Siqueira CM, Homsani F, Veiga VF, Lyrio C, Mattos H, Passos SR, et al. Homeopathic medicines for prevention of influenza and acute respiratory tract infections in children: blind, randomized, placebo-controlled clinical trial. Homeopathy. 2016 Feb;105(1):71-7. https://doi.org/10.1016/j.homp.2015.02.006.

7. Cajueiro APB, Goma EP, Santos HAM, Almeida-Rodrigues I, Toma HK, Araújo SM, et al. Homeopathic medicines cause Th1 predominance and induce spleen and megakaryocytes changes in BALB/c mice infected with Leishmania infantum. Cytokine. 2017 Jul;95:97101. https://doi.org/10.1016/j.cyto.2017.02.010.

8. Mondal J, Das J, Shah R, Khuda-Bukhsh AR. A homeopathic nosode, Hepatitis C 30 demonstrates anticancer effect against liver cancer cells in vitro by modulating telomerase and topoisomerase II activities as also by promoting apoptosis via intrinsic mitochondrial pathway. J Integr Med. 2016 May;14(3):209-18. https://doi.org/10.1016/S20954964(16)60251-0.

9. Holandino C, Harduim R, Veiga VF, Garcia S, Zacharias CR. Modeling physical chemical properties of high dilutions: an electrical conductivity study. Int J High Dilution Res. 2008;7(25):165-73.

10. Holandino C, Leal FD, Barcellos B, Campos MA, Oliveira R, Veiga VF, et al. Mechanical versus handmade succussions: a physical chemistry comparison. In: Bonamin LV, editor. Signal and Images. Heidelberg: Springer; 2008. p. 37-48.

11. Garcia S, Harduim RC, Homsani F, Zacharias CR, Kurster R, Holandino C. Physical chemical and citotoxic evaluation of highly diluted solutions of Euphorbia tirucalli L. prepared through the fifty milesimal homeopathic method. Int J High Dilution Res. 
ISSN 2179-6750

2010;9(31):63-73.

12. Homsani F, Barbosa GM, Silva LFM, Siqueira CM, Santos LH, El-Bacha T, et al. Evaluation of alterations in adhesion rates of Candida albicans and in respiration cellular induced by Candida albicans type RC nosodes. In: Proceedings of the XXVII GIRI Symposium; 2013 September 21-23; Florence; São Paulo: UNIFESP. p. 152-3.

13. Homsani F, Larossa CP, Gonçalves F, Avellar D, Santos AL, Garcia S, et al. Physicalchemical and microbiological stability of biotherapy Candida albicans RC in different potencies In: Proceedings of the XXV GIRI Symposium and VIII Congresso Brasileiro de Farmácia Homeopática; 2011 setmbro 4-7; Foz do Iguaçu; São Paulo: Unifesp. p. 155-6.

14. Costa BGB, Clark R, Homsani F, Holandino C. Evaluation of physicochemical features of Zincum metallicum homeopathic solutions In: Proceedings of the XXVII GIRI Symposium; 2013 Sptember 3-4; Bem (Switzerland); São Paulo: Unifesp. p. 85.

15. Elia V. Physico-chemical properties of perturbed water: facts and enigmas. Int J High Dilution Res. 2012;11(40):110-2.

16. Elia V, Napoli E, Germano R. The 'Memory of Water': an almost deciphered enigma. Dissipative structures in extremely dilute aqueous solutions. Homeopathy. 2007 Jul;96(3):163-9. https://doi.org/10.1016/j.homp.2007.05.007.

17. Davenas E, Beauvais F, Amara J, Oberbaum M, Robinzon B, Miadonna A, et al. Human basophil degranulation triggered by very dilute antiserum against IgE. Nature. 1988;333(6176):816-8.

18. Rey L. Can low-temperature thermoluminescence cast light on the nature of ultra-high dilutions? Homeopathy. 2007 Jul;96(3):170-4. https://doi.org/10.1016/j.homp.2007.05.004.

19. Montagnier L, Aissa J, Del Giudice E, Lavallee C, Tedeschi A, Vitiello G. DNA waves and water. J Phys Conf Ser. 2011;306:012007. https://doi.org/10.1088/1742-6596/306/1/012007.

20. Bellavite P, Marzotto M, Olioso D, Moratti E, Conforti A. High-dilution effects revisited. 1. Physicochemical $\quad$ aspects. Homeopathy. 2014 Jan;103(1):4-21. https://doi.org/10.1016/j.homp.2013.08.003.

21. Oliveira SM, Oliveira CC, Abud APR, Guimarães FSF, Di Bernardi RP, Coletto ELO, et al. Mercurius solubilis: actions on macrophages. Homeopathy. 2011Oct;100(4):228-36. https://doi.org/10.1016/j.homp.2011.05.005.

22. Bonamin LV, Sato C, Zalla Neto R, Morante G, Cardoso TN, Santana FR. Immunomodulation of Homeopathic Thymulin $5 \mathrm{CH}$ in a BCG-induced granuloma model. 330 
ISSN 2179-6750

Evid Based Complement Alternat Med. 2013;2013:686018. https://doi.org/10.1155/2013/686018

23. Molski M. Quasi-quantum phenomena: the key to understanding homeopathy. Homeopathy. 2010 Apr;99(2):104-12. https://doi.org/10.1016/j.homp.2009.11.009.

24. Klein SD, Sanding A, Baumgartner S, Wolf U. Differences in median ultraviolet light transmissions of serial homeopathic dilutions of copper sulfate, Hypericum perforatum, and sulfur. Evid Based Complement Alternat Med. 2013;2013:370609. https://doi.org/10.1155/2013/370609.

25. Anagnostatos GS, Vithoulkas G, Garzonis P, Tavouxoglou C. A working hypothesis for homeopathic microdiluted remedies. $\mathrm{Br}$ Homeopath J. 1992;81(1):67. https://doi.org/10.1016/S0007-0785(05)80310-2.

26. Anagnostatos GS. Small water clusters clathrates in the preparation process of homoeopathy. In: Endler PC, Schulte, J, editors. Ultra High Dilution. Dordrecht: Kluwer Acad. Publ; 1994. p. $121-8$.

27. Smith CW. Electromagnetic and magnetic vector potential bioinformation and water. Homeopathy. 2015 Oct;104(4):301-4. https://doi.org/10.1016/j.homp.2015.08.006.

28. Chaplin MF. A proposal for the structuring of water. Biophys Chem. 2000 Jan 24;83(3):21121. https://doi.org/10.1016/S0301-4622(99)00142-8.

29. Voeikov VL. The possible role of active oxygen in the memory of water. Homeopathy. 2007 Jul;96(3):196-201. https://doi.org/10.1016/j.homp.2007.05.003

30. Chaplin MF. The Memory of water: an overview. Homeopathy. 2007 Jul;96(3):143-50. https://doi.org/10.1016/j.homp.2007.05.006

31. Chikramane PS, Suresh AK, Bellare JR, Kane SG. Extreme homeopathic dilutions retain starting materials: a nanoparticulate perspective. Homeopathy. 2010 Oct;99(4):231-42. https://doi.org/10.1016/j.homp.2010.05.006.

32. Upadhyay RP, Nayak C. Homeopathy emerging as nanomedicine. Int J High Dilution Res. 2011 Dec;10(37):299-310.

33. Vybíral B, Vorácek P. Long term structural effects in water: autothixotropy of water and its hysteresis. Homeopathy. 2007 Jul;96(3):183-8. https://doi.org/10.1016/j.homp.2007.03.007.

34. Demangeat JL. Gas nanobubbles and aqueous nanostructures: the crucial role of dynamization. Homeopathy. 2015 Apr;104(2):101-15. https://doi.org/10.1016/j.homp.2015.02.001.

35. Lee HM, Kim KS. Dynamics and structural changes of small water clusters on ionization. J 
ISSN 2179-6750

Comput Chem. 2013 Jul 5;34(18):1589-97. https://doi.org/10.1002/jcc.23296.

36. Anick DJ, Ives JA. The silica hypothesis for homeopathy: physical chemistry. Homeopathy. 2007 Jul;96(3):189-95. https://doi.org/10.1016/j.homp.2007.03.005

37. Roy R, Tiller WA, Bell I, Hoover MR. The structure of liquid water: novel insights from materials research: potential relevance to homeopathy. Mater Res Innov. 2005;9(4):98-103. https://doi.org/10.1080/14328917.2005.11784911.

38. Bellavite P, Magnani P, Zanolin E, Conforti A. Homeopathic doses of Gelsemium sempervirens improve the behavior of mice in response to novel environments. Evid Based Complement Alternat Med. 2011;2011:362517. https://doi.org/10.1093/ecam/nep139.

39. Chirumbolo S, Brizzi M, Ortolani R, Vella A, Bellavite P. Inhibition of CD203c membrane up-regulation in human basophils by high dilutions of histamine: a controlled replication study. Inflamm Res. 2009 Nov;58(11):755-64. https://doi.org/10.1007/s00011-009-0044-4. 